Asian Social Work Journal (ASWJ), Volume 5, Issue 2, (page 6 - 16), 2020

DOI: https://doi.org/10.47405/aswj.v5i2.134

\begin{tabular}{|c|c|}
\hline & Asian Social Work Journal (ASWJ) \\
$\substack{\text { ASIAN SOCIAL WORK } \\
\text { JOURAL } \\
\text { (ASW) }}$ & Volume 5, Issue 2, July 2020 \\
& e-ISSN : 0128-1577 \\
& Journal home page: \\
& www.msocialwork.com \\
\hline
\end{tabular}

\title{
Health Problems, Misconceptions and Challenges: Experiences of Slum Dweller Adolescent Girls in Bangladesh
}

\author{
Muhammed Muazzam Hussain ${ }^{1}$ \\ ${ }^{1}$ Shahjalal University of Science and Technology, Sylhet, Bangladesh \\ Correspondence: Muhammed Muazzam Hussain (muazzam777@yahoo.com)
}

\begin{abstract}
The aim of the paper is to know the health condition of adolescent girls living in the slum areas of Sylhet city, Bangladesh, understand their physical health problems, mental health issues and identify misconceptions and challenges etc. The study was based on primary sources of data; however the secondary data e.g. official documents, books, journals, policy papers etc. were used to conduct the study. The study followed qualitative research approach and the case study method to generate sufficient primary data. The study findings show that adolescent girls of slum areas face multiple health challenges including physical health problems, mental ill health, poverty etc. They suffer from different types of diseases e.g. headache, fever, cough and cold, gastric ulcer, diarrhea, asthma and allergy, jaundice, skin diseases etc. The study also highlights some misconceptions on menstruation which added another dimension of health challenges that they face in their daily lives. In addition, family poverty prevents them in taking nutritious food which is manifested in malnutrition, iron deficiency and other health complexities. Therefore, some key areas of policy recommendations are outlined for improving their health condition.
\end{abstract}

Keywords: health problem, misconception, adolescent girl, slum

\section{Introduction}

Bangladesh is characterized as the most densely populated countries of the world having 162.7 million populations with 1103 people per square kilometer (BBS, 2018). The people living in urban areas and cities constitute 35.86 percent of the total population (Statista, 2017). The Census of Slum Areas and Floating Population (2014) reveals that a total of 22,32,114 population live in the slum areas which is accounted for 6.33 per cent of the total urban population of the country. The number of males were $11,43,337$, females were 10,86,337 and transgender community known as hizra were 1,852 (BBS, 2015). Adolescence is the transition period from childhood to adulthood and is widely recognized as a time of great importance due to vulnerabilities linked with this period in terms of biological, physical, social, and environmental considerations (Blum and Bastus, 2012). It has been regarded as a period of human growth and development which includes those persons between 10 and 19 years of age (WHO, 2014). At present, the adolescent cohort among the global population is the largest ever, adolescents aged 10-19 years comprise about 18\% of the global population (UNICEF, 2011). In Bangladesh, the adolescents represent more than 20 per cent of the country's total population i.e. approximately 36 million (BDHS, 2014, BBS, 2015). Various studies suggest that people including the adolescents living in slums are facing miserable condition and most of them are undernourished, illiterate and do not have knowledge about the nutritional value of foods. Due to poor sanitation and congested living they often suffer from diseases like diarrhea, dysentery, typhoid, dengue and pneumonia (cited in Rahman and 
DOI: https://doi.org/10.47405/aswj.v5i2.134

Alam, 2015). It is also revealed that majority of adolescent girls did not have any details idea about menstruation until they got their first experience. In majority cases it was found that parents or guardians of adolescent girls thought it is a matter of shy and taboo involved with discussing that aspect (Tasneem, 2013). Some other studies also demonstrate that adolescent school girls faced various challenges during menstruation e.g. maintaining special secrecy, misconception and misunderstanding, lack of using sanitary pads due to high cost and family poverty etc. which put their reproductive health at risk and prone to diseases (Chandra \& Patel, 2017, Muhit et al., 2013, Barkat et al, 2003).

The Bangladesh Multiple Indicator Cluster Survey (2012-2013) shows that early childbearing is a common incident in Bangladesh where about one in four women e.g. 24.4 per cent of women in age 2024 years have experienced the birth of their first baby before the age of 18 . Recently, the government of Bangladesh has adopted a National Adolescent Health Strategy (2017-2030) to address the overall health needs of adolescents by taking a broad and holistic understanding of the concept of health (MCH Services Unit, 2016). It is worthwhile to investigate their health condition i.e. physical and mental health problems and identify misconceptions, challenges etc. so that appropriate measures could be taken to improve their condition and wellbeing. Therefore, the objectives of the study were: to know the health condition of adolescent girls living in the slum areas of Sylhet city, understand their physical and mental health problems and identify misconceptions and challenges etc.

\section{Theoretical framework}

A general understanding of the people's health condition i.e. both physical health problems and mental health aspects require the consideration of different theoretical approaches and models within health care. Peoples' health condition including misconceptions and challenges that they experiences can be better understood on the basis of the following theoretical viewpoints: the ecological model, biomedical approach, health belief model, social cognitive theory, the theory of planned behavior and social determinants of health perspective etc. The ecological model focus on multiple factors that influence health behaviors including intrapersonal or individual factors, (e.g. knowledge, attitudes, beliefs, and personality) institutional and organizational factors (e.g. the rules, regulations, policies as well as the informal structures that constrain or promote healthy behaviors) community factors (e.g. formal or informal social norms etc.) that can limit or enhance healthy behaviors; public policy factors, including local, state, and federal policies and laws that regulate or support health actions and practices for disease prevention control, and management (Knudson, 2015). However, this approach is very important as it is comprehensive in nature and helps us to get a holistic understanding on the health condition, problems and challenges faced by adolescent girls of slum areas, it is very difficult in practice to clarify all of the components and address the challenges involved with those factors. The bio-medical approach (BMA) focus on understanding the epidemiological and biomedical factors related to disease with the objective to eliminate and control disease through screening and treatment (Holman, 1992). The positive outcome of biomedical is well recognized and is manifested in the recent improvement in life expectancy, but the dominance of this approach may ignore the socio- economic and cultural factors that shape the living condition, health care practices and the general wellbeing of individuals including adolescent girls. By contrast, the health belief model (HBM) focus on individual beliefs, perception on health conditions and predict individual health-related behaviors including perceived threat to sickness or disease, it's consequence, potential benefits of action and perceived barriers to action etc. Understanding health belief, perception and attitude is also important to understand health condition and misconception of individuals, but it would provide only a partial picture of the challenges faced by the adolescent girls and to improve their current health and wellbeing. On the contrary, the social cognitive theory (SCT) and theory of planned behavior (PB) upholds individual experiences, the actions of others, and environmental factors on individual health behaviors (Viner et al. 2012). However those theories provides opportunities for social support through exploring expectations, self-efficacy and utilizing observational learning etc., it can hardly address structural barriers e.g. social inequality, low income, lack of adequate housing, water and sanitation etc. that affect on health condition of people. The social determinants of health (SDH) perspective focuses on the material disadvantage, social inequality, social structures within which people live and the experiences they get etc. shape their wellbeing (WHO, 2008). Therefore, the SDH framework supports a policy shift from illness based discourses to wellness 
DOI: https://doi.org/10.47405/aswj.v5i2.134

based discourses that emphasize prevention of illness and chronic diseases as well as people's positive social experiences of better health outcomes. Therefore, the current study was guided by SDH approach as it was thought more suitable for a better understanding and exploring the experiences of adolescent girls, their perception, physical health problems, mental health issues, misconception and challenges etc.

\section{Methodology}

The study was based on primary sources of data; however the secondary data e.g. official documents, books, journals, policy papers etc. were used to conduct the study. The study followed qualitative research approach and the case study method to generate sufficient primary data. The in-depth interviews were undertaken with semi-structured interview schedule to collect necessary information to address the research objectives. In addition, two focus group discussions were arranged with six to eight participants in each group to substantiate the interview data.

\section{The study area, population and sampling}

The Sylhet City Corporation (SCC) was selected purposively as the area of study. All of the adolescent girls living in the slums of SCC were considered as the population of study. According to the BBS report (2015), there are 671 slums in Sylhet city with a number of total population are 50,129. The Sylhet city was chosen as it is different from the capital city Dhaka where majority large sums are prevailed and majority studies have been conducted there. By contrast, the Sylhet city corporation is a growing urban area and slum settlements are very distinct in nature e.g. much smaller than other major cities of the country in terms of population size, location and ownership which are known as 'Koloni' rather than 'Basthi'. Three (03) slums were purposively selected on the basis of density of slum dwellers for recruiting research participants. Twenty one (21) adolescences girls were selected from those three (03) slums under the ward no. 9, 13 and 15 and two FGDs were conducted with another two slums under the ward no. 3 and 5 of the SCC. The following inclusion criteria were selected to recruit the research participants:

i. To be included in the study the participant should girl under the age of 12 to 19 years old and live in the slums, known as 'Koloni' for at least one year.

ii. Literate and illiterate, married and unmarried girls of the above mentioned criteria could participate in the study.

\section{Data collection}

Semi-structure interview guidelines were prepared to collect necessary data to address the research objectives. In many cases, questions were open ended with a number of sub questions, which were substituted for the following three main research questions:

i. What is the physical health condition of adolescent girls and what types of health problems do they face?

ii. What is the mental health condition of them or do they face any mental health challenge?

iii. Do they have any misconception or face any challenges to maintain their current health?

On an average the duration of in depth interviews were 45 minutes to 1 hour and the FGDs were 2 hours to 2 hours and 30 minutes in length. The researcher along with two data collectors carried out the interviews and conducted the FGDs to understand health issues of adolescent girls, their health belief, mental condition etc.

\section{Ethical aspects}

The researchers followed the ethical guidelines offered by Miles and Huberman (1994). Participation in the study was entirely voluntary and relied on the ethical principle of informed consent. At the start of interview and FGD, the study purpose and nature was explained. They were asked to choice whether 
DOI: https://doi.org/10.47405/aswj.v5i2.134

they can participate or not to participate and those who wanted to participate, the written consent was taken from those who could write and verbal consent was taken from those who did not know how to put signature.

\section{Data analysis}

The in depth interviews and FGDs were audio recorded with the permission of the respondents. The verbatim recordings were transcribed and translated into English by the researcher and two research assistants. The data were read, re-read and taken for open coding and thematic analysis. From the original data codes were generated in terms of the various concepts and issues and then selective coding was conducted to systematically analyze the data and identify the most prominent themes. Data saturation was achieved after at the end in depth interview when the same data were repeating and no new information were found. The 'iterative' analysis process was followed and a particular attention was given to choosing and using the quotations which is most relevant to the specific issue (Chen, 2013).

\section{The Findings}

\section{Health problems and challenges}

The study findings reveal that majority of the adolescent girls living in the slums had suffered one or more types of physical health problems including fever, cough and headache, etc. The following narratives point out the issue:

"I suffer from fever, cough and headache six to seven times in a year. I feel very weak and do not take proper treatment due shortage of money. My mother usually goes to pharmacy and brings some medicine for me but it does provide a temporary relief." (Age, 17 years)

"I had suffered from malaria fever two months ago. I felt dizziness and the whole body was shaking. I slept like an unconscious person. There were none to look after me because my parents went to work and it was a nightmare for me" (Age, 15 years)

"Malaria is almost a common disease in this slum. Once I had suffered from fever for 15 days at a stretch which broken down my health. Now I feel very weak and tired without doing any work." (FGD, 01)

The study findings also show that majority of the adolescent girls of slum suffer from water borne diseases e.g. diarrhea, jaundice, skin diseases etc. They face additional difficulties as there are no private washrooms, adequate clean water and toilet facilities and consequently they feel embarrassed. The following excerpts echo the issue:

"I suffer many times from stomach related diseases e.g. diarrhea and dysentery. I face difficulties in using washroom or toilet because we do not have privacy and sometimes need to wait long time in queue and feel shame." (Age, 16 years)

"I am suffering from different types of skin problems e.g. rush, itching, black spot in the body etc. Since, we sometimes use impure pond water in taking bath or washing cloth and utensils, we have been affected by those diseases." (Age, 14 years)

"Many people in our slum including me suffer from dermatitis and eczema, itching etc. As I need to work in the kitchen long hours and use impure water my skin condition has been deteriorated." (Age, 15 years) 
DOI: https://doi.org/10.47405/aswj.v5i2.134

The study findings also reveal that a significant number of adolescent girls of slum areas have been suffering from respiratory diseases. The family poverty and overcrowded housing condition create a big challenge to them. The following narratives echo the issues:

"I am a patient with allergy and breathing shortage. I feel much hardship to live in the overcrowded house where all of our family members bound live in a large room." (Age, 15 years)

"I have been suffering from asthma problem. Since we are poor and do not have appropriate warm cloth and blankets we face difficulties in winter season e.g. pain in chest and breathing shortage etc. and it seems that I am going to die. " (Age, 14 years)

The study findings reveal that a significant number of adolescent girls are suffering from gastric and ulcer diseases, malnutrition and anemia and face some challenges e.g. poverty of the family, irregularity in eating, stay long hours without taking food, eating excessive spicy food etc. that increases their health vulnerability. The following excerpts echo the issue:

"I am suffering from gastric problem and pain in stomach. I go to school by taking tea, biscuit or 'chira' (dried rice) as breakfast and my mother cannot manage other food for breakfast. I stay long time in school and take lunch at the evening. This kind of food habit creates major health challenge for me which I don't like; but nothing to do." (Age, 15 years)

"I am suffering from malnutrition and anemia. However, the doctor advised me to take nutritious food e.g. eggs and milk etc., but my parents cannot mange those foods for me due to poverty." (Age, 17 years)

"Every time after menstruation I feel very weak and face difficulties to do common daily tasks of family e.g. cooking food and take care of my baby. The doctor identifies iron and calcium deficiency and suggests me to take milk, meat, egg, fruits to remove health problems, but how could we manage?" (FGD, 02)

\section{Menstruation issue: Misconception and challenges}

The study demonstrate out that a large number of adolescent girls have some misconceptions about menstruation issue. They do not have much clear idea on it as a natural health aspect of female person; rather they feel shame on it. The following excerpts echo the issue:

"I just knew that menstruation is a shameful disease of women. Before starting menstruation, I felt serious abdominal pain and became afraid when I saw bleeding." (Age, 15 years)

"I had no idea about the menstruation. So when my first menstruation started I became tensed and nervous. I couldn't share this with anybody due to shame and I did just whipping." (Age, 17 years)

The study findings also demonstrate that adolescent girls in slum areas cannot afford sanitary pads during menstruation. They use rags, commonly known as 'nekra' which are usually made by old clothes e.g. piece of cotton saris and dresses. The study also point out that they face some difficulties in cleaning rags because, there is a lack of private place to change and clean it. They thought it would be a matter of shame and other male persons or boys would tease them and could make fun or use slang languages to them. The following narratives raised the issue: 
Asian Social Work Journal (ASWJ), Volume 5, Issue 2, (page 6 - 16), 2020

DOI: https://doi.org/10.47405/aswj.v5i2.134

"Cleaning rags is a big challenge for me as I cannot find a private place to change and wash it. In this koloni, we have only five toilets and four bathrooms for thirty families. I feel shame to go there with taking nekra and soap." (Age, 15 years)

"I can't clean the rags at day time because both male and female members of the slum take bath in the same tube-well. I have to wait for night to change and wash rags." (Age, 14 years)

"I feel shy to bring 'nekra' and soap with me when I go to common washroom or toilet as other male members of slum also use that washroom or toilet. If they look it, I will feel much shame. " (Girl, 13 Years)

The schools going adolescent girls also face challenges during menstruation period to change pads or rags at the time of school. In many cases it is found that there are no separate wash room or toilet facilities in schools for boys and girls students. The following narratives clarify the issue:

"At the time of my menstruation I don't go to school regularly as there are no specific places for girls to change the rag." (Age, 13 years)

"I face difficulties to change or wash nekra in school as there is no separate washroom for girls. It is embarrassing for me." (Age, 15 years)

\section{Mental health issues and challenges}

The study findings also show that majority of the married adolescent girls face tension, stress, anxiety and depression as major mental health issues; but they are not aware that these problems require treatment. The following narratives raise the issue:

"I have consulted with doctor about my sleeping problem but do not say about tension and depression. I don't think it's a matter of discussion, rather a personal matter." (Age, 17 years)

"I always feel tensed about the different issues of my family. As I can't share my problems with anyone and I get depressed. But I don't think it's a disease require treatment; rather it will be removed in course of time." (FGD, 02)

The study findings also demonstrate that menstruation period also effects on the mental health condition of adolescent girls. They sometimes feel depressed, angry and behave roughly with family members and friends. The following narratives clarify the situation:

"Excessive bleeding and abdominal pain are major problems during my menstruation which continue 10 or 12 days. I feel stress and sometimes get hot temper and become angry quickly." (FGD, 01)

"I feel headache and become tensed during the menstruation period. Sometimes my mood became off, I remain quiet and don't like to talk with others." (Age, 15 years)

The study findings also demonstrate that marriage during adolescence period creates lots of mental pressure for some married adolescent girls. The following excerpts echo the issue:

"My health condition has been deteriorated after my marriage. As I loved someone and my parents forced me to marriage with another person, I couldn't eat and sleep properly after my marriage. (Age, 15 years) 
DOI: https://doi.org/10.47405/aswj.v5i2.134

"I am struggling hard to cope with my husband. My husband is not a good person, do not love me; rather sometimes beat me for minor reasons. It creates a mental pressure for me and causes sleep disturbances." (Age, 16 years)

"My husband is a very hot tempered person. If I disagree with his any opinion, he often starts beating me. I am not happy with him and couldn't leave him because of my son. So I feel depressed." (FGD, 02)

"I live in a joint family and as a daughter-in-law I need to do most of the household work e.g. cook food for all the members and stay in the kitchen for a long time. I don't get mental peace for that." (Married AG, Age, 17 years)

\section{Discussion}

The major objectives of the study was to know the physical and mental health condition of slum dweller adolescent girls and identify health problems, misconceptions and challenges etc. However, this study provide some valuable insights on the health condition e.g. physical health problems, menstruation issue, mental health challenges, it could not be generalized due to limited coverage and small number of samples selected by using purposive sampling procedure.

\section{Health challenges: Psycho- social aspects}

The study findings reveal that majority of the adolescent girls living in the slums had suffered one or more types of physical health problems including fever, cough and headache, etc. However they feel very weak, they do not take proper treatment due shortage of money. Their parents usually go to medical vendor or pharmacy for bringing some medicine which provide a temporary relief. Another challenge they face include lack of family caring as they belong to the poor category, their mother and father need to work to run the family. It is evident from the study that they face additional difficulties e.g. lack of caring by parents when they become ill. However, some other studies (e.g. Ainul et al., 2017) identified the scarcity of public services available for adolescents; this study findings point out limitation of caring opportunities available inside the family.

The study findings reveal that a significant number of adolescent girls are suffering from gastric and ulcer diseases, malnutrition and anemia and face some challenges e.g. poverty of the family, irregularity in eating, stay long hours without taking food, eating excessive spicy food etc. that increases their health vulnerability. The study demonstrates that a significant number of adolescent girls are bound to go to school by taking tea, biscuit or 'chira' (dried rice) as breakfast. They need to stay long time in school and take lunch at the evening. This kind of food habit creates major health challenge for them. The study findings also reveal that majority of them suffer from malnutrition and anemia. Due to poverty, their parents cannot afford nutritious food e.g. eggs, milk, fruits etc. These study findings are consistent with some other research findings which pointed out that the risk of low birth weight (LBW) and preterm delivery is particularly high among iron-deficient anemic adolescents. The risk of LBW is significantly higher in young adolescents aged 10-14 years. It was further clarified that the risk of dying from pregnancy-related causes is twice as high for women aged 15-19 years and five times higher for girls aged 10-14 years as for women aged 20-29 years (WHO, 2007).

The study findings also reveals that majority of slum dweller adolescent girls suffer from water borne diseases e.g. diarrhea, jaundice, skin diseases etc. They face additional difficulties as there are no private washrooms, adequate clean water and toilet facilities etc. Due to lack of washroom and toilet, they need to wait long time in the queue and eventually they feel shame and embarrassed. The other health challenges include using impure pond water in taking bath or washing cloth and utensils which causes skin problems e.g. dermatitis and eczema, rush, itching, black spot in the body etc. The findings resonate with another study which clarified that adverse living conditions of urban slums e.g. poor access to health services, hygiene condition, water and sanitation system etc. constitute a big challenge for them (Ahmed et al., 2010). 
DOI: https://doi.org/10.47405/aswj.v5i2.134

The study findings also reveal that a significant number of adolescent girls of slum areas have been suffering from respiratory diseases. The family poverty and overcrowded housing condition create a big challenge to them. Since, majority of them are poor and do not have appropriate warm clothes and blankets, they face difficulties during winter season. The study resonate with other studies which pointed out that majority of the adolescent girls living in slum areas are suffering from various infectious and chronic diseases and the environmental degradation e.g. water and air pollution increases the risk of causing diseases (Rashid, 2009, Izutsu, 2006).

\section{Menstruation related misconceptions}

The study demonstrate out that a large number of adolescent girls have some misconceptions about menstruation issue. They do not have much clear idea about it. They do not think it as a natural health aspect of women; rather feel shame for it. They perceive it as a shameful disease of women and should not discuss with other person. They feel fear, anxiety and nervousness which reduce their mental happiness.

The study findings also demonstrate that adolescent girls in slum areas cannot afford sanitary pads during menstruation. They use rags, commonly known as 'nekra' which are usually made by old clothes e.g. piece of cotton 'saris' and dresses. The study also point out that they face some difficulties in cleaning rags because, there is a lack of private place to change and clean it. They fear that it would be a matter of shame if other male persons or boys would see it. Since, majority of them are bound to use common toilet, washroom, they feel shame to go there with 'nekra' and soap. Because, they thought people might tease them and could make fun or use slang languages to them. The findings mirror similarity with other studies which revealed that majority them do not follow hygienic health care practices during their menstruation period due to poverty and lower socio-economic condition of family (Suha, 2013, Werven, 2010).

The school going slum dweller adolescent girls also face challenges during menstruation period to change pads or rags at the time of school. In many cases it is found that there are no separate wash room or toilet facilities in schools for girls students. Therefore, they sometimes drop classes in the school during menarche. The study findings also resonate with other study which revealed that adolescent school girls cannot afford using sanitary pads during menstruation due to financial hardship. They also face some challenges e.g. lack of private bath and latrine, adequate water supply, lack of gender unfriendly school etc. which bound them to get absent from the school and social activities (Muhit et al., 2013).

\section{Mental health challenges}

The study findings also show that majority of the married adolescent girls face tension, stress, anxiety and depression as major mental health problems. But the reality is that they do not think it as problems which require treatment. It is evident from the study that some of them consulted with doctor about sleeping problem but do not talk about tension, anxiety and depression. The study findings also demonstrate that menstruation period also effects on the mental health condition of adolescent girls. They sometimes feel depressed, angry and behave roughly with family members and friends. Some of them feel headache and become tensed during the menstruation period. These findings are consistent with some previous studies which pointed out that 22.9 per cent of socially disadvantaged urban slum children and adolescents had some form of psychiatric and behavior disorder (Hossain et al. 2014, Liu et al., 2010, Naila et al., 2009, Kaltiala et al., 2003).

The study findings also demonstrate that marriage during adolescence period creates lots of mental pressure for some married adolescent girls. It is revealed that health condition of AG has been deteriorated after marriage. Not all but in some cases it is found that their parents arrange marriages without their consent which creates mental pressure and stress for them. They face difficulties to cope up with their new life events e.g. relationship with husband, parents in-laws and other responsibilities. The study broke a new ground for potential risk of domestic violence which might happen to slum dweller adolescent girls and causes mental health problems for them. For example, living in a joint 
DOI: https://doi.org/10.47405/aswj.v5i2.134

family and as a daughter-in-law to perform household work e.g. cook food for all the members and stay in the kitchen for a long time etc. creates potential mental health risk for them. The findings are also consistent with country's demographic profile which showed that one-quarter of girls age 15 through19 years are already married; and by the age of 19, 70 per cent of them have had a child (Cortez et al., 2014). Similar other studies also suggest prevalence of poor mental health among the slum dwellers and pointed out spatial associations between mental health and housing quality, sanitation, income generation, environmental health knowledge, education etc. (Gruebner et al., 2011).

\section{Conclusion and Policy Implications}

The study findings suggest that adolescent girls of slum areas are one of the significant segments of population in our country. They face multiple challenges and vulnerability in terms of their physical health problems, mental health complexities, age and gender sensitivity and poverty etc. They are deprived of basic needs and suffer from different types of physical health problems and diseases e.g. headache, fever, cough and cold, gastric ulcer, diarrhea, asthma and allergy, jaundice, skin diseases etc. Family poverty prevents them in taking nutritious food which is manifested in malnutrition, iron deficiency and anemia and causes gastric and acidity problems. They are less conscious about their health condition and feel shy to share their health related problems with others. It is evident from the study that their health related issues do not get priority to the family members and relatives and there remain misconception among parents or guardians that menstruation related health problems will be automatically removed after marriage. Since, adolescent pregnancy, preterm birth and early child bearing etc. are often regarded major health concerns for adolescent girls of Bangladesh, more rigorous qualitative and quantitative researches could be done to investigate whether there is a link between the misconception of parents and child marriage in the slum areas. Moreover, the married adolescent girls face additional difficulties to maintain family responsibilities e.g. household activities including cooking, washing clothes, look after babies etc. which create lots of mental pressure on them. Due to ignorance they do not think it as mental health problems and do not consult with physician which reduces mental peace and stability. Since, they are the future mother of nation, their health and wellbeing should be given much priority in the current development discourse. It is often argued that mental health care system in Bangladesh faces multifaceted challenges such as lack of public mental health facilities, scarcity of skilled workforce, inadequate financial resource allocation and social stigma etc. (Islam and Biswas, 2015). Priority should be given to focus on the mental health issue of adolescent girls, such as giving advice regarding the menstrual health, control of emotion, reduce mental stress, tension and anxiety etc. It is argued that being a professional today means becoming interprofessional (Meads and Ashcraft, 2005:3). Therefore, public and private service providers, academicians, social workers, psychologists, nutritionists, local power structure should be involved in service planning and implementation of programs. Service providers should also recognize the importance of service user's belief, perception and cultural aspects and ensure their participation in decision making and designing of projects undertaken for them.

\section{References}

Ahmed, S. M., Hossain, A., Khan, M. A., Mridha, M. K., Alam, A., Choudhury, N., . . Bhuiya, A. (2010). Using formative research to develop MNCH programme in urban slums in Bangladesh: experiences from MANOSHI, BRAC. BMC public health, 10(1), 663.

Ainul, S., Bajracharya, A., Reichenbach, L., \& Gilles, K. (2017). Adolescents in Bangladesh: A situation analysis of programmatic approaches to sexual and reproductive health education and services. Washington, DC \& Dhaka, Bangladesh: Population Council.

Bangladesh Bureau of Statistics (BBS), \& United Nations Children's Fund (UNICEF). (2015). Accessed on

$03 / 03 / 2019$. http://203.112.218.65:8008/WebTestApplication/userfiles/Image/LatestReports/MICS_201213.pdf.

Barkat, A., \& Majid, A. (2003). Adolescent Reproductive Health in Bangladesh: Status, Policies, Programs and issues. Dhaka: USAID. 
BBS. (2014). Dhaka: Bangladesh Bureau of Statistics, Statistics and Informatics Division, Ministry of Planning.

BBS. (2015). Census of Slum Areas and Floating Population 2014. Dhaka: Bangladesh Bureau of Statistics, Statistics and Informatics Division, Ministry of Planning, Government of the People's Republic of Bangladesh.

BBS. (2018). Bangladesh Sample Vital Statistics 2017. Dhaka: Bangladesh Bureau of Statistics, Planning division, Ministry of Planning, The Government of the People's Republic of Bangladesh.

BDHS. (2014). Bangladesh Demographic and Health Survey Dhaka: National Institute of Population, Research and Training (NIPORT), Ministry of Health and Family Welfare.

Chandra-Mouli, V., \& Patel, S. V. (2017). Mapping the knowledge and understanding of menarche, menstrual hygiene and menstrual health among adolescent girls in low-and middle-income countries. Reproductive health, 14(1), 30.

Cortez, R., Hinson, L., \& Petroni, S. (2014). Adolescent Sexual and Reproductive Health in Dhaka's Slums, Bangladesh: World Bank Group. Retrieved from: http://documents.worldbank.org/curated/en/712551468014450655/pdf/912910BRI0Adol00Box 385330B00PUBLIC0.pdf Accessed: 04-03-2019.

Gruebner, O., Khan, M. H., Lautenbach, S., Müller, D., Kraemer, A., Lakes, T., \& Hostert, P. (2011). A spatial epidemiological analysis of self-rated mental health in the slums of Dhaka. International Journal of Health Geographics, 10(1), 36.

Hossain, M. D., Ahmed, H. U., Chowdhury, W. A., Niessen, L. W., \& Alam, D. S. (2014). Mental disorders in Bangladesh: a systematic review. BMC psychiatry, 14. doi:10.1186/s12888-0140216-9

Islam, A., \& Biswas, T. (2015). Mental Health and the Health System in Bangladesh: Situation Analysis of a Neglected Domain. American Journal of Psychiatry and Neuroscience, 3(4), 57 62. doi:10.11648/j.ajpn.20150304.11

Izutsu, T., Tsutsumi, A., Islam, A. M., Kato, S., Wakai, S., \& Kurita, H. (2006). Mental health, quality of life, and nutritional status of adolescents in Dhaka, Bangladesh: Comparison between an urban slum and a non-slum area. Social Science \& Medicine, 63(6), 1477- 1488.

Islam, M. R., \& Faruque, C. J. (Eds.) (2016 \& 2017). Qualitative research: Tools and techniques. USA: Farwood Publishing and Dhaka: AH Development Publishing House.

Kaltiala-Heino, R., Marttunen, M., Rantanen, P., \& Rimpelä, M. (2003). Early puberty is associated with mental health problems in middle adolescence. Social Science \& Medicine, 57(6), 10551064.

Khan, N. Z., Ferdous, S., Islam, R., Sultana, A., Durkin, M., \& McConachie, H. (2009). Behaviour Problems in Young Children in Rural Bangladesh. Journal of tropical pediatrics, 55(3), 177182. doi:https://doi.org/10.1093/tropej/fmn108.

Knudson, A. (2015). Rural Health Promotion and Disease Prevention Toolkit. Rural Health Information Hub. Retrieved from: https://www.ruralhealthinfo.org/toolkits/healthpromotion/2/theories-and-models/reasoned-action Accessed on: 01-04-2018.

Liu, X., Yang, Y., Yuan, P., Zhang, X., Han, Y., Cao, Y., \& Xiong, G. (2010). A study of the relationship between mental health and menstrual abnormalities in female middle school students from post-earthquake Wenchuan. Bioscience trends, 4(1).

M., M., \& A., H. (1994). Qualitative data analysis: An expanded source book. Thousand Oaks CA: Sage Publications.

MCH Services Unit. (2016). National Strategy for Adolescent Health 2017-2030. Dhaka: Directorate General of Family Planning Ministry of Health and Family Welfare Government of the People's Republic of Bangladesh Retrieved from: http://coastbd.net/wpcontent/uploads/2017/07/National-Strategy-for-Adolescent-Health-2017-2030-Final-Full-Book21-06-17.pdf Date: 04-03-2019.

Muhit, I. B., \& Chowdhury, S. T. (2013). Menstrual Hygiene condition of Adolescent School girls at Chittagong Division in Bangladesh. International Journal of Scientific and Technology Research, 2(6).

Pryer, J. A., \& Rogers, S. (2006). Epidemiology of undernutrition in adults in Dhaka slum households. European journal of clinical nutrition, 60(7), 815 . 
DOI: https://doi.org/10.47405/aswj.v5i2.134

Rahman, M. H., \& Alam, S. S. (2015). Nutritional Status of Children in Slums of Dhaka, Bangladesh. J Nutr Food Sci, 5, 425. doi:10.4172/2155-9600.1000425

Rashid, S. F. (2007). Durbolota (weakness), chinta rog (worry illness), and poverty: explanations of white discharge among married adolescent women in an urban slum in Dhaka, Bangladesh. Medical Anthropology Quarterly, 21(1), 108-132.

Rashid, S. F. (2009). Strategies to reduce exclusion among populations living in urban slum settlements in Bangladesh. Journal of health, population, and nutrition, 27(4), 574.

Statista. (2017). Retrieved from https://www.statista.com/statistics/455782/urbanization-inbangladesh/ Date: 03-03-2019

Suha, S. M. (2013). Adolescent Girls in Urban Slum: Environmental Health Perspectives. The International Journal of Social Sciences, 9(28).

UNICEF. (2011). The state of the world's children 2011-adolescence: an age of opportunity: United Nations Children's Fund (UNICEF).

Viner, R. M., Ozer, E. M., Denny, S., Marmot, M., Resnick, M., Fatusi, A., \& Currie, C. (2012). Adolescence and the social determinants of health. The lancet, 379(9826), 1641-1652.

Warven, I. V. (2010). Health and hygiene School Program Initiative for Adolescents in Dhaka. The Journal of Gender and Water, 1(34-36).

World Health Organization. (2007). Adolescence pregnancy: Unmet needs and undone Needs Available https://apps.who.int/iris/bitstream/handle/10665/43702/9789241595650_eng.pdf;jsessionid=44F 8809C7EFDBF3590F81A5F4CADFECB?sequence=1. Accessed: 25-02-2018.

WHO Commission on Social Determinants of Health, \& World Health Organization. (2008). Closing the gap in a generation: health equity through action on the social determinants of health: Commission on Social Determinants of Health final report. World Health Organization. 